\title{
FIVE-SIDED MECHANISM, DETERMINATION OF ACCELERATION
}

\author{
Izolda POPA-MÜLLER ${ }^{1}$, István PAPP ${ }^{2}$
}

Sapientia - Hungarian University of Transylvania, Faculty of Technical and Human Sciences, Department of Mechanical Engineering, Târgu-Mureș, Romania

${ }^{1}$ ipmuller@ms.sapientia.ro

${ }^{2}$ pappistvan1944@yahoo.com

\begin{abstract}
The aim of this paper is the presentation of the general form of the constraint equations necessary to calculate the accelerations occuring on a five sided spatial mechanism. Using these equations the computing of the accelerations for any part of any plain or spatial mechanism will be possible.

The constraint equations of the acceleration are obtained by computing the time derivatives of the velocity equations (which in general form are given by [1] and [2]) followed by the correspondent grouping of the unknowns.
\end{abstract}

Keywords: thermoelectric materials, recent research, nano structures, dopping, substitution.

\section{Presentation of the five-sided spatial mechanism}

The elements of the five-sided mechanism are (Figure 1.):

1 - fuselage;

2 - crankshaft;

3 - crank;

4 - connecting member;

5 - swing arm.

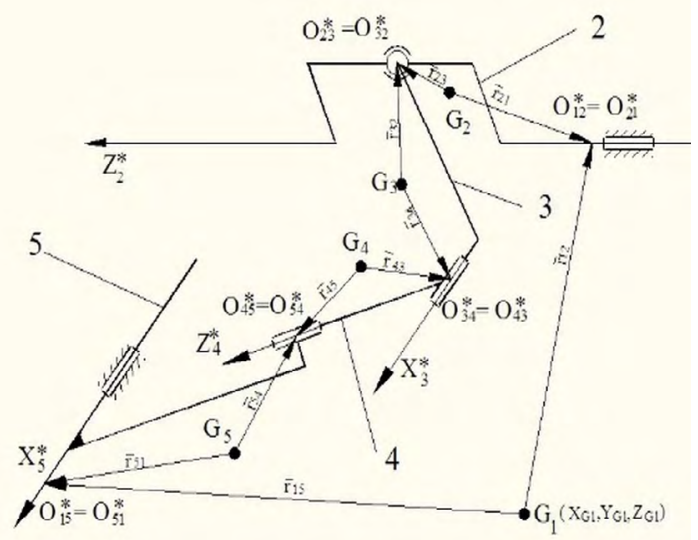

Figure 1. Five-sided spatial mechanism
The program is written for an external fixed system in which the coordinates of the mass centre of the machine fuselage are given $X_{G i}, Y_{G i}, Z_{G i}$ values. The coordinates of $O_{i j}^{*}$ points relative to of the centers of gravity masses of any member part of the mechanism are alwaysremain constants during the motion and they are determined by the vectors $r_{i j}$ or $r_{i j} I$.

\section{The constraint equations of the accel- eration}

The computing of the unknowns we built-up a system containing sixteen constraint equations. The leading parameter of the mechanism is the rotation angle $\Psi_{2}$ of the crankshaft. The general form of the constraint equations must be deduced from the general equations of a cylindrical and a spherical joint.The cylindrical joint bind the fuselage 1 and the crancshaft 2 . Thus, the constraint equations of this rotary joint and the related acceleration are the followings: 
$\ddot{X}_{G 2}-x_{21} \cdot \dot{\psi}_{2}^{2} \cdot \alpha_{12}\left(\psi_{2}, \theta_{2}, \phi_{2}\right)-y_{21} \cdot \dot{\psi}_{2}^{2} \cdot \beta_{12}\left(\psi_{2}, \theta_{2}, \phi_{2}\right)-z_{21} \cdot \dot{\psi}_{2}^{2} \cdot \gamma_{12}\left(\theta_{2}, \psi_{2}\right)=0$

$\ddot{Y}_{G 2}-x_{21} \cdot \dot{\psi}_{2}^{2} \cdot \alpha_{22}\left(\psi_{2}, \theta_{2}, \phi_{2}\right)-y_{21} \cdot \dot{\psi}_{2}^{2} \cdot \beta_{22}\left(\psi_{2}, \theta_{2}, \phi_{2}\right)-z_{21} \cdot \dot{\psi}_{2}^{2} \cdot \gamma_{22}\left(\theta_{2}, \psi_{2}\right)=0$

In the same way are given the constraint equations for the spherical joint that binds the crankshaft 2 and the crank 3 :

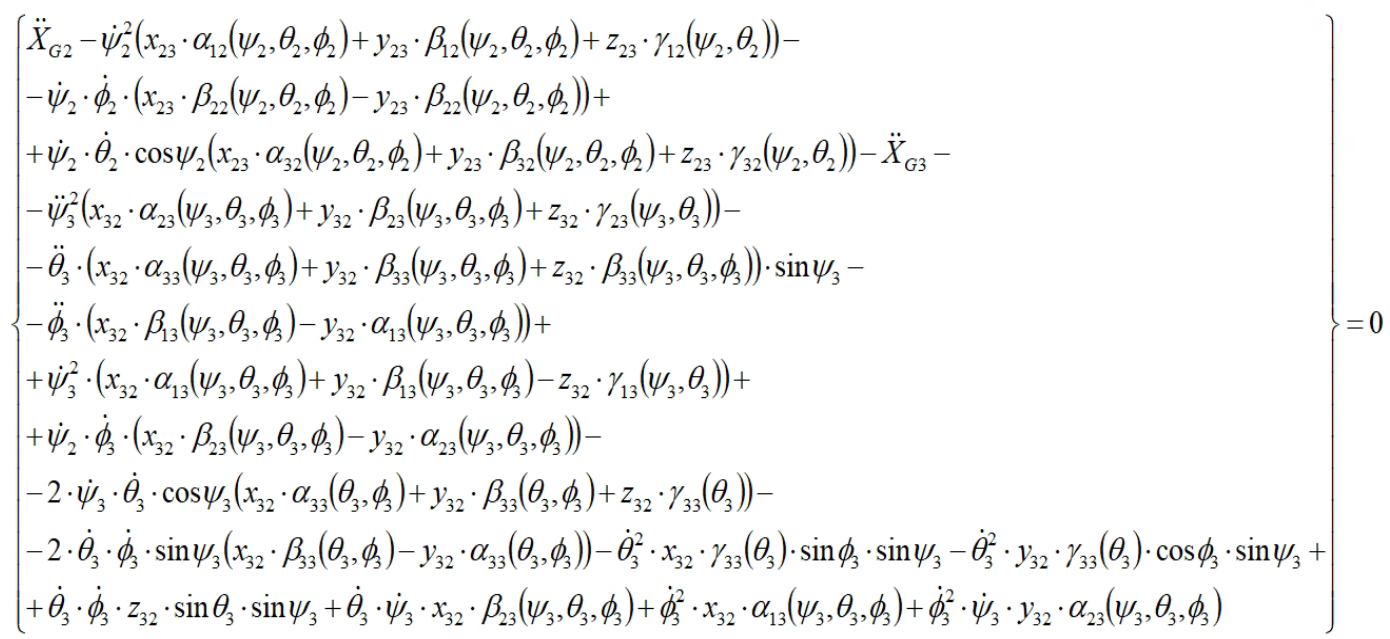

$$
\begin{aligned}
& \left(\ddot{Y}_{G 2}-\dot{\psi}_{2}^{2}\left(x_{23} \cdot \alpha_{22}\left(\psi_{2}, \theta_{2}, \phi_{2}\right)+y_{23} \cdot \beta_{22}\left(\psi_{2}, \theta_{2}, \phi_{2}\right)+z_{23} \cdot \gamma_{22}\left(\psi_{2}, \theta_{2}, \phi_{2}\right)\right)-\right. \\
& -\dot{\psi}_{2} \cdot \dot{\phi}_{2} \cdot\left(y_{23} \cdot \alpha_{12}\left(\psi_{2}, \theta_{2}, \phi_{2}\right)-x_{23} \cdot \beta_{12}\left(\psi_{2}, \theta_{2}, \phi_{2}\right)\right)+ \\
& +\dot{\psi}_{2} \cdot \dot{\theta}_{2} \cdot \sin \psi_{2}\left(x_{23} \cdot \alpha_{32}\left(\psi_{2}, \theta_{2}, \phi_{2}\right)+z_{23} \cdot \gamma_{32}\left(\psi_{2}, \theta_{2}, \phi_{2}\right)\right)-\dot{\psi}_{2} \cdot \sin \psi_{2} \cdot y_{23} \cdot \beta_{32}\left(\psi_{2}, \theta_{2}, \phi_{2}\right)- \\
& -\ddot{Y}_{G 3}-\ddot{\psi}_{3}\left(x_{32} \cdot \alpha_{13}\left(\psi_{3}, \theta_{3}, \phi_{3}\right)+y_{32} \cdot \beta_{13}\left(\psi_{3}, \theta_{3}, \phi_{3}\right)+z_{32} \cdot \gamma_{13}\left(\psi_{3}, \theta_{3}\right)\right)+ \\
& +\ddot{\theta}_{3} \cdot\left(x_{32} \cdot \alpha_{33}\left(\theta_{3}, \phi_{3}\right)+y_{32} \cdot \beta_{33}\left(\psi_{3}, \theta_{3}, \phi_{3}\right)+z_{32} \cdot \beta_{33}\left(\psi_{3}, \theta_{3}, \phi_{3}\right)\right) \cdot \cos \psi_{3}- \\
& -\ddot{\phi}_{3} \cdot\left(x_{32} \cdot \beta_{23}\left(\psi_{3}, \theta_{3}, \phi_{3}\right)-y_{32} \cdot \alpha_{23}\left(\psi_{3}, \theta_{3}, \phi_{3}\right)\right)+\dot{\psi}_{3}^{2} \cdot \dot{\phi}_{3} \cdot \alpha_{23}\left(\psi_{3}, \theta_{3}, \phi_{3}\right) \cdot \beta_{13}\left(\psi_{3}, \theta_{3}, \phi_{3}\right) \cdot x_{32}- \\
& -\dot{\psi}_{3} \cdot \dot{\theta}_{3} \cdot \sin \psi_{3}\left(x_{32} \cdot \alpha_{33}\left(\theta_{3}, \phi_{3}\right)+y_{32} \cdot \beta_{33}\left(\theta_{3}, \phi_{3}\right)+z_{32} \cdot \gamma_{33}\left(\theta_{3}\right)\right)+ \\
& +\dot{\psi}_{3}^{2}\left(y_{32} \cdot \beta_{23}\left(\psi_{3}, \theta_{3}, \phi_{3}\right)+z_{32} \cdot \gamma_{23}\left(\psi_{3}, \theta_{3}\right)\right)+2 \dot{\psi}_{3} \cdot \dot{\phi}_{3} \cdot y_{32}\left(\psi_{3}, \theta_{3}\right) \cdot \alpha_{13}\left(\psi_{3}, \theta_{3}, \phi_{3}\right)+ \\
& +2 \cdot \dot{\theta}_{3} \cdot \dot{\phi}_{3} \cdot \cos \psi_{3}\left(x_{32} \cdot \beta_{33}\left(\theta_{3}, \phi_{3}\right)-y_{32} \cdot \alpha_{33}\left(\theta_{3}, \phi_{3}\right)\right)+\dot{\theta}_{3} \cdot \dot{\phi}_{3} \cdot\left(y_{32} \cdot \alpha_{13}\left(\psi_{3}, \theta_{3}, \phi_{3}\right)-x_{32} \cdot \beta_{13}\left(\psi_{3}, \theta_{3}, \phi_{3}\right)\right)+ \\
& +\ddot{\phi}_{3}^{2} \cdot\left(x_{32} \cdot \alpha_{23}\left(\psi_{3}, \theta_{3}, \phi_{3}\right)+y_{32}\left(\psi_{3}, \theta_{3}\right) \cdot \beta_{23}\left(\psi_{3}, \theta_{3}, \phi_{3}\right)\right)+\dot{\theta}_{3}^{2} \cdot y_{32}\left(\psi_{3}, \theta_{3}\right) \cdot \gamma_{33}\left(\theta_{3}\right) \cdot \cos \phi_{3} \cdot \cos \psi_{3}+ \\
& +\dot{\theta}_{3}^{2} \cdot x_{32} \cdot \gamma_{33}\left(\theta_{3}\right) \cdot \sin \phi_{3} \cdot \cos \psi_{3}-\dot{\theta}_{3} \cdot \dot{\phi}_{3} \cdot z_{32} \cdot \sin \theta_{3} \cdot \cos \psi_{3}- \\
& -\dot{\theta}_{3} \cdot \dot{\psi}_{3} \cdot\left(x_{32} \cdot \alpha_{33}\left(\theta_{3}, \phi_{3}\right)+y_{32} \cdot \beta_{33}\left(\theta_{3}, \phi_{3}\right)+z_{32} \cdot \gamma_{33}\left(\theta_{3}, \phi_{3}\right)\right) \cdot \sin \psi_{3} \\
& =0 \\
& \left\{\begin{array}{l}
\ddot{Z}_{G 3}+\ddot{\theta}_{3} \cdot \sin \phi_{3} \cdot \gamma_{33}\left(\theta_{3}\right) \cdot x_{32}+\ddot{\theta}_{3} \cdot \cos \phi_{3} \cdot \gamma_{32}\left(\psi_{3}, \theta_{3}\right) \cdot \gamma_{33}\left(\theta_{3}\right)-\ddot{\theta}_{3} \cdot \sin \theta_{3} \cdot z_{32}+ \\
+\ddot{\phi}_{3} \cdot\left(x_{32} \cdot \beta_{33}\left(\theta_{3}, \phi_{3}\right)-y_{32} \cdot \alpha_{33}\left(\theta_{3}, \phi_{3}\right)\right)-\dot{\theta}_{3} \cdot \dot{\phi}_{3} \cdot \sin \theta_{3} \cdot \sin \phi_{3} \cdot x_{32}-\dot{\theta}_{3} \cdot \dot{\phi}_{3} \cdot \sin \theta_{3} \cdot \cos \phi_{3} \cdot y_{32}-\dot{\theta}_{3}^{2} \cdot \sin \theta_{3} \cdot z_{32}+ \\
+2 \dot{\theta}_{3} \cdot \dot{\phi}_{3} \cdot \cos \phi_{3} \cdot x_{32} \cdot \gamma_{33}\left(\theta_{3}\right)-2 \dot{\theta}_{3} \cdot \dot{\phi}_{3} \cdot \sin \phi_{3} \cdot y_{32} \cdot \gamma_{33}\left(\theta_{3}\right)-\dot{\phi}_{3}^{2} \cdot\left(x_{32} \cdot \alpha_{33}\left(\theta_{3}, \phi_{3}\right)+y_{32} \cdot \beta_{33}\left(\theta_{3}, \phi_{3}\right)\right)
\end{array}\right\}=0
\end{aligned}
$$


These are followed by the writing-up of the equations of acceleration between the connecting member 4 and the swing arm 5:

$\ddot{X}_{G 4}+\ddot{\theta}_{4} \cdot\left(x_{45} \cdot \alpha_{34}\left(\theta_{4}, \phi_{4}\right)+y_{45} \cdot \beta_{34}\left(\theta_{4}, \phi_{4}\right)+z_{45} \cdot \gamma_{34}\left(\theta_{4}\right)\right) \cdot \sin \psi_{4}+\ddot{\phi}_{4} \cdot\left(x_{45} \cdot \beta_{14}\left(\psi_{4}, \theta_{4}, \phi_{4}\right)-y_{45} \cdot \alpha_{14}\left(\psi_{4}, \theta_{4}, \phi_{4}\right)\right)+$ $+\dot{\theta}_{4} \cdot\left(x_{45} \cdot \dot{\alpha}_{34}\left(\theta_{4}\right)+y_{45} \cdot \dot{\beta}_{34}\left(\theta_{4}, \phi_{4}\right)+z_{45} \cdot \dot{\gamma}_{34}\left(\theta_{4}\right)\right) \cdot \sin \psi_{4}+\dot{\phi}_{4} \cdot\left(x_{45} \cdot \dot{\beta}_{14}\left(\psi_{4}, \theta_{4}, \phi_{4}\right)-y_{45} \cdot \dot{\alpha}_{14}\left(\psi_{4}, \theta_{4}, \phi_{4}\right)\right)-$

$-\ddot{\theta}_{5} \cdot\left(x_{54} \cdot \alpha_{35} \cdot\left(\theta_{5}, \phi_{5}\right)+y_{54} \cdot \beta_{35}\left(\theta_{5}, \phi_{5}\right)+z_{54} \cdot \gamma_{35}\left(\theta_{5}\right)\right) \cdot \sin \psi_{5}-$

$-\dot{\theta}_{5} \cdot\left(x_{54} \cdot \dot{\alpha}_{35} \cdot\left(\theta_{5}, \phi_{5}\right)+y_{54} \cdot \dot{\beta}_{35}\left(\theta_{5}, \phi_{5}\right)+z_{54} \cdot \dot{\gamma}_{35}\left(\theta_{5}\right)\right) \cdot \sin \psi_{5}=0$

$\ddot{Y}_{G 4}-\ddot{\theta}_{4} \cdot\left(x_{45} \cdot \alpha_{34}\left(\theta_{4}, \phi_{4}\right)+y_{45} \cdot \beta_{34}\left(\theta_{4}, \phi_{4}\right)+z_{45} \cdot \gamma_{34}\left(\theta_{4}\right)\right) \cdot \cos \psi_{4}+\ddot{\phi}_{4} \cdot\left(x_{45} \cdot \beta_{24}\left(\psi_{4}, \theta_{4}, \phi_{4}\right)-y_{45} \cdot \alpha_{24}\left(\psi_{4}, \theta_{4}, \phi_{4}\right)\right)+$ $-\dot{\theta}_{4} \cdot\left(x_{45} \cdot \dot{\alpha}_{34}\left(\theta_{4}\right)+y_{45} \cdot \dot{\beta}_{34}\left(\theta_{4}, \phi_{4}\right)+z_{45} \cdot \dot{\gamma}_{34}\left(\theta_{4}\right)\right) \cdot \cos \psi_{4}+\dot{\phi}_{4} \cdot\left(x_{45} \cdot \dot{\beta}_{24}\left(\psi_{4}, \theta_{4}, \phi_{4}\right)-y_{45} \cdot \dot{\alpha}_{24}\left(\psi_{4}, \theta_{4}, \phi_{4}\right)\right)-\ddot{Y}_{G 5}+$ $+\ddot{\theta}_{5} \cdot\left(x_{54} \cdot \alpha_{35} \cdot\left(\theta_{5}, \phi_{5}\right)+y_{54} \cdot \beta_{35}\left(\theta_{5}, \phi_{5}\right)+z_{54} \cdot \gamma_{35}\left(\theta_{5}\right)\right) \cdot \cos \psi_{5}+$

$+\dot{\theta}_{5} \cdot\left(x_{54} \cdot \dot{\alpha}_{35} \cdot\left(\theta_{5}, \phi_{5}\right)+y_{54} \cdot \dot{\beta}_{35}\left(\theta_{5}, \phi_{5}\right)+z_{54} \cdot \dot{\gamma}_{35}\left(\theta_{5}\right)\right) \cdot \cos \psi_{5}=0$

$\ddot{Z}_{G 4}+\ddot{\theta}_{4} \cdot\left(x_{45} \cdot \gamma_{34}\left(\theta_{4}\right) \cdot \sin \phi_{4}+y_{45} \cdot \gamma_{34}\left(\theta_{4}\right) \cdot \cos \phi_{4}-z_{45} \cdot \sin \theta_{4}\right)+$

$+\ddot{\phi}_{4} \cdot\left(x_{45} \cdot \beta_{34}\left(\theta_{4}, \phi_{4}\right)-y_{45} \cdot \alpha_{34}\left(\theta_{4}, \phi_{4}\right)\right)+$

$+\dot{\theta}_{4} \cdot\left(x_{45} \cdot \dot{\gamma}_{34}\left(\theta_{4}\right) \cdot \sin \phi_{4}+y_{45} \cdot \dot{\gamma}_{34}\left(\theta_{4}, \phi_{4}\right) \cdot \cos \phi_{4}-z_{45} \cdot \dot{\theta}_{4} \cdot \cos \theta_{4}\right)+$

$+\dot{\theta}_{4} \cdot \dot{\phi}_{4}\left(x_{45} \cdot \dot{\gamma}_{34} \cdot \cos \phi_{4}-y_{45} \cdot \dot{\gamma}_{34}\left(\theta_{4}, \phi_{4}\right) \cdot \sin \phi_{4}\right)+\dot{\phi}_{4} \cdot\left(x_{45} \cdot \dot{\beta}_{34}\left(\theta_{4}, \phi_{4}\right)-y_{45} \cdot \dot{\alpha}_{34}\left(\theta_{4}, \phi_{4}\right)\right)-\ddot{Z}_{G 5}-$

$-\ddot{\theta}_{5} \cdot\left(x_{54} \cdot \gamma_{35} \cdot\left(\theta_{5}\right) \cdot \sin \phi_{5}+y_{54} \cdot \gamma_{35}\left(\theta_{5}\right) \cdot \cos \phi_{5}-z_{54} \cdot \sin \theta_{5}\right)-$

$\left.-\dot{\theta}_{5} \cdot\left(x_{54} \cdot \dot{\gamma}_{35} \cdot\left(\theta_{5}\right) \cdot \sin \phi_{5}+y_{54} \cdot \dot{\gamma}_{35}\left(\theta_{5}\right) \cdot \sin \phi_{5}\right)-z_{54} \cdot \dot{\theta}_{5} \cos \theta_{5}\right)=0$

$\ddot{\theta}_{4} \cdot\left(\gamma_{34} \cdot \gamma_{14}^{0} \cdot \sin \phi_{4}+\gamma_{34} \cdot \gamma_{24}^{0} \cdot \cos \phi_{4}+\gamma_{34} \cdot \sin \theta_{4}\right)+\dot{\theta}_{4} \cdot\left(\dot{\gamma}_{34} \cdot \gamma_{14}^{0} \cdot \cos \phi_{4}-\dot{\gamma}_{34} \cdot \gamma_{24}^{0} \cdot \sin \phi_{4}+\dot{\gamma}_{34} \cdot \sin \theta_{4}\right)+$ $+\ddot{\phi}_{4} \cdot\left(\beta_{34} \cdot \gamma_{14}^{0}-\alpha_{34} \cdot \gamma_{24}^{0}\right)+\dot{\phi}_{4} \cdot\left(\dot{\beta}_{34} \cdot \gamma_{14}^{0}-\dot{\alpha}_{34} \cdot \gamma_{24}^{0}\right)-\ddot{\phi}_{5} \cdot\left(\beta_{35} \cdot \gamma_{15}^{0}-\alpha_{35} \cdot \gamma_{25}^{0}\right)-\dot{\phi}_{5} \cdot\left(\dot{\beta}_{35} \cdot \gamma_{15}^{0}-\dot{\alpha}_{35} \cdot \gamma_{25}^{0}\right)-$ $-\ddot{\theta}_{5} \cdot\left(\gamma_{35} \cdot \gamma_{15}^{0} \cdot \sin \phi_{5}+\gamma_{35} \cdot \gamma_{25}^{0} \cdot \cos \phi_{5}+\gamma_{35} \cdot \sin \theta_{5}\right)-\dot{\theta}_{5} \cdot\left(\dot{\gamma}_{35} \cdot \gamma_{15}^{0} \cdot \cos \phi_{5}-\dot{\gamma}_{35} \cdot \gamma_{25}^{0} \cdot \sin \phi_{5}+\dot{\gamma}_{35} \cdot \sin \theta_{5}\right)=0$

The equations of constraint between parts 4 and 5 (swing arm and connecting part) are formally identical with those related to elements 3 and 4 (crank and connecxting part). Tese resume to the transléation along axes $\mathrm{X}$. Thus, acceleration equations for the The swing arm are the followings:

$\ddot{Y}_{G 5}-\ddot{\theta}_{5} \cdot\left(x_{51} \cdot \alpha_{35}+y_{51} \cdot \beta_{35}+z_{51} \cdot \gamma_{35}\right) \cdot \cos \psi_{5}-\dot{\theta}_{5} \cdot\left(x_{51} \cdot \dot{\alpha}_{35}+y_{51} \cdot \dot{\beta}_{35}+z_{51} \cdot \dot{\gamma}_{35}\right) \cdot \cos \psi_{5}=0$

$\ddot{Z}_{G 5}+\ddot{\theta}_{5} \cdot\left(x_{51} \cdot \gamma_{35}\left(\theta_{5}\right) \cdot \sin \phi_{5}+y_{51} \cdot \gamma_{35}\left(\theta_{5}\right) \cdot \cos \phi_{5}-z_{51} \cdot \sin \theta_{5}\right)-\dot{\theta}_{5}^{2} \cdot \sin \theta_{5} \cdot \sin \phi_{5} \cdot x_{51}-$

$-\dot{\theta}_{5}^{2} \cdot \sin \theta_{5} \cdot \cos \phi_{5} \cdot y_{51}-\dot{\theta}_{5}^{2} \cdot \cos \theta_{5} \cdot z_{51}=0$

The matrix of the 16 unknowns of the problem are presented in the following transposed vertex:

$\left[Q_{i}\right]^{T}=\left(\ddot{X}_{G 2}, \ddot{Y}_{G 2}, \ddot{X}_{G 3}, \ddot{Y}_{G 3}, \ddot{Z}_{G 3}, \ddot{X}_{G 4}, \ddot{Y}_{G 4}, \ddot{Z}_{G 4}, \ddot{Y}_{G 5}, \ddot{Z}_{G 5}, \ddot{\psi}_{3}, \ddot{\theta}_{3}, \ddot{\phi}_{3}, \ddot{\theta}_{4}, \ddot{\phi}_{4}, \ddot{\theta}_{5}\right)$

The occuring derivatives are given in the specific form of the formulas [3] 170, p. 5,179, 164, 5,172 are generally formulated. 


\section{Conclusions}

Using the constraint equations, accelerations in any plane or spatial mechanism can be computed without the use of the explicite particular motion equations.

The method described in above offers the best way for exactly computing of, the accelerations related to the central inertia axes relative to their own or the external fixed frame.

Scientific results can be used to calculate accelerations of any lever mechanism or robot, as well as to develop new and effective laboratory practices in educational use.

\section{References}

[1] Papp I.: Contributions to Dynamic Balancing of Spatial Mechanisms. PhD Thesis, Cluj-Napoca, 2000.

[2] Papp I., Popa-Müller I.: Kinematic Recognition with constraint equations. Research Contract No. 1298, Sapientia Foundation Research Programs Institute, 2005.

[3] Papp I.: Theory of Mechanisms. Scientia Publishing, Cluj-Napoca, ISBN 978-973-1970-29-5, 2010. 